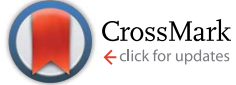

Cite this: Energy Environ. Sci., 2015, 8, 876

Received 23rd July 2014

Accepted 23rd December 2014

DOI: $10.1039 / c 4 e e 02314 e$

www.rsc.org/ees

\section{A sensitivity analysis to assess the relative importance of improvements in electrocatalysts, light absorbers, and system geometry on the efficiency of solar-fuels generators}

\author{
Yikai Chen, Shu Hu, Chengxiang Xiang* and Nathan S. Lewis*
}

A sensitivity analysis has been performed for a variety of generic designs for solar-fuels generators. The analysis has revealed the relative importance of reductions in the overpotentials of electrocatalysts, of improvements in the materials properties of light absorbers, and of optimization in the system geometry for various different types of solar-fuels generators, while considering operation at a range of temperatures as well as under a variety of illumination intensities including up to 10 -fold optical concentration.

\section{Introduction}

Most concepts for a solar-fuels generator include components for light absorption and charge separation, electrocatalysts for one or both of the half-reactions involved in the production of fuels from $\mathrm{H}_{2} \mathrm{O}$ or from $\mathrm{H}_{2} \mathrm{O}$ and $\mathrm{CO}_{2}$, and a membrane or other physical separation barrier to ensure separation of the products. ${ }^{1-3}$ All of these envisioned system components are the basis for active areas of research, with the goal of improving the activity, stability, and mutual compatibility of the various components for use in a fully operational, efficient, robust, intrinsically safe, scalable, demonstration of a solar-fuels generator. ${ }^{\mathbf{1 - 1 1}}$

In the discipline of systems engineering, a sensitivity analysis is a routine, critical tool used to identify the main levers, i.e. the components of the system for which improvements in performance will have the most impact on improving the performance of the system as a whole. ${ }^{12}$ In general, a sensitivity analysis can only beneficially be performed when a system design is in hand, because the architecture of the system will play a significant, if not dominant, role in the outcome and will determine the inputs and outputs of the system-based sensitivity analysis. For example, different sensitivity analyses would

Beckman Institute, Kavli Nanoscience Institute, and Joint Center for Artificial Photosynthesis, 210 Noyes Laboratory, 127-72, Division of Chemistry and Chemical Engineering, California Institute of Technology, Pasadena, CA 91125, USA. E-mail: cxx@caltech.edu; nslewis@caltech.edu

\begin{abstract}
Broader context
A solar-driven water-splitting cell is generally comprised of light absorbers, electrocatalysts, membrane separators and an electrolyte solution in a specific system geometry. The overall solar-to-hydrogen conversion efficiency of such a system depends on the performance and materials properties of all the individual components as well as the design of the system. Significant research efforts are being devoted to improving the performance of all of the system components, yet some improvements will result in larger gains in the overall system efficiency than others. We describe herein a sensitivity analysis of the solar-to-hydrogen conversion efficiency with respect to the materials properties of light absorbers, electrocatalysts, and the geometric design parameters, for a series of specific but generic designs for solar-fuels generators. Such a sensitivity analysis provides a quantitative framework within which to assess the gains in system performance that can be attained as a result of improving, relative to the current state-of-the-art, the performance of different components of the system, and provides a useful framework for setting a forward R\&D agenda for such systems.
\end{abstract}

be needed to ascertain the key levers in optimizing the flight speed or flight time of a jet-powered, fixed-wing aircraft relative to optimizing the speed or flight time of a helicopter.

The theoretical, and practically realizable, efficiencies of a solar-driven water-splitting device based on theoretical materials properties, or on current state-of-the-art materials and components, have not been presented in the literature for a specific system design concept in hand. ${ }^{\mathbf{1 3 - 1 5}}$ Recently, several generic systems-level concepts for solar-fuels generators have been presented ${ }^{\mathbf{1 6 - 2 0}}$ in sufficiently specific detail to enable a meaningful sensitivity analysis to identify the key levers that will produce the largest performance improvements within the overall design space of the systems of interest. The state-of-theart performance values of many classes of electrocatalysts for key reactions of interest, including the hydrogen-evolution reaction (HER), the oxygen-evolution reaction (OER), and possible $\mathrm{CO}_{2}$-reduction reactions $\left(\mathrm{CO}_{2} \mathrm{RR}\right)$, have also recently been compiled and documented. ${ }^{11,21,22}$ Additionally, the state-ofthe-art performance of individual light absorbers, as well as the performance of combinations of light absorbers for use in 
tandem structures, have been recently reviewed. ${ }^{1}$ Extensive modeling and simulation efforts, using validated multi-physics modeling approaches, have also been performed recently on a variety of system geometries and for a variety of operating temperatures, illumination intensities, and optical concentration factors. ${ }^{\mathbf{1 6 - 2 0}}$

We report herein a "one factor at a time" sensitivity anal$y \operatorname{sis}^{23-25}$ for several types of generic designs of solar-fuels generators. In this study, the sensitivity of the efficiency of the generation of solar fuels to the properties of the various system components was evaluated as a function of the total overpotential of the electrocatalysts for a variety of light absorbers having a range of band gaps and having varying materials quality. An additional sensitivity analysis has been performed to determine the key levers for improving the efficiency of solarfuels generation over a range of operating temperatures and under concentrated illumination. The sensitivity analysis clearly shows the extent to which improvements, relative to the state-of-the-art, in the overpotential of the electrocatalysts, in the system geometry and design, in the properties of the light absorbers, and in the properties of the membranes will provide gains in overall attainable system efficiency. In this respect, the sensitivity analysis serves to survey the possible system design space and highlights areas that will have the largest impact on improving the performance of the system as a whole.

\section{Modeling}

\section{Device designs}

The generic design of the solar-driven water-splitting device investigated herein contained a photoabsorber component, electrocatalyst layers for the OER and for the HER, a membrane separator, and liquid electrolytes. The generic design includes several specific cell constructs. The detailed geometric parameters of these cell constructs have been described previously. ${ }^{16-18,20}$ The photoabsorber component contained a tandem-junction photoelectrochemical cell, in which $100 \%$ of the incident solar photon flux arrives at the photoabsorber surface in either an unconcentrated, planar design or in a concentrated design coupled with a $10 \times$ optical solar concentrator. The optical obscuration due to the liquid electrolyte, electrocatalyst layers, and membrane separators were neglected in the calculation. The OER and HER electrocatalyst layers were located directly on the top and the bottom of the anode and cathode sides of the photoabsorber component, respectively, in which the geometric surface area of the HER catalyst and OER catalyst were identical and were bounded to the geometric area of the tandem photoabsorber. The membrane separator was employed to provide the required ion-transport pathways between the cathode and anode compartments as well as to provide effective barriers to the crossover of products. The liquid electrolyte contained strong acid or strong base (e.g., $1 \mathrm{M}$ $\mathrm{H}_{2} \mathrm{SO}_{4}(\mathrm{aq})$ or $1 \mathrm{M} \mathrm{KOH}(\mathrm{aq})$ ), which produced negligible $\mathrm{pH}$ gradients at the surfaces of the electrocatalysts under operating conditions. The transport of ions through the membrane and in the liquid electrolyte was the source of any additional resistive losses in the device.
Although other solar-fuel generators, e.g., $\mathrm{CO}_{2}$-reduction reactors, contain some components in common with the solardriven water-splitting device described above, the specific cell designs and operational constraints of a $\mathrm{CO}_{2}$-reduction reactor could vary significantly from those of a water-splitting reactor, due to different system-level constraints. Moreover, depending on the specific reduction products, the thermodynamic potentials for other fuel-forming reactions can vary significantly from those of water splitting. Thus, the modeling and simulation results and associated sensitivity analysis described herein are only explicitly directed towards, and applicable, to solar-driven water-splitting systems of the general design classes evaluated herein.

\section{Solar-to-hydrogen (STH) conversion efficiency}

The operating current density, $J_{\mathrm{op}}$, of an integrated set of light absorbers arranged in a tandem configuration was calculated by the following equation:

$$
V_{\text {op }}\left(J_{\text {op }}\right)=\phi_{0}+\eta_{\mathrm{OER}}\left(J_{\mathrm{op}}\right)+\left|\eta_{\mathrm{HER}}\left(J_{\mathrm{op}}\right)\right|+R_{\text {eff }}\left(J_{\mathrm{op}}\right)
$$

where $V_{\text {op }}\left(J_{\text {op }}\right)$ is the current-voltage relationship of the tandem photoabsorbers, $\phi_{0}$ is the thermodynamic potential for the water-splitting reaction, $R_{\text {eff }}$ is the effective transport resistance in the membrane separator and the liquid electrolyte, and $\eta_{\text {OER }}\left(J_{\text {op }}\right)$ and $\eta_{\text {HER }}\left(J_{\text {op }}\right)$ are the overpotentials for the OER and HER, respectively.

The STH conversion efficiency, $\eta_{\mathrm{STH}}$, is defined as:

$$
\Phi_{\mathrm{STH}}=\frac{1.23(V) \times J_{\mathrm{op}}\left(\mathrm{mA} \mathrm{cm}^{-2}\right)}{S\left(\mathrm{~mW} \mathrm{~cm} \mathrm{~cm}^{-2}\right)}
$$

with $J_{\text {op }}$ the operating photocurrent density $\left(\mathrm{mA} \mathrm{cm}^{-2}\right)$ and $S$ the total incident solar irradiance $\left(\mathrm{mW} \mathrm{cm}^{-2}\right)$.

\section{Shockley-Queisser limit for light absorbers}

The ideal limiting case, i.e. the Shockley-Queisser (S-Q) limit, in which the current-voltage relationship for a tandem photoabsorber is determined by use of a detailed-balance calculation, is obtained when the current density at the operating photovoltage is equal to the sum of the incident solar radiation $\left(J_{\mathrm{ph}}\right)$ and the thermal radiation $\left(J_{\mathrm{th}}\right)$ minus the radiative emission $\left(J_{\text {rad }}\right):^{26}$

$$
J=J_{\mathrm{ph}}+J_{\mathrm{th}}-J_{\mathrm{rad}}
$$

$J_{\mathrm{ph}}, J_{\text {th }}$ and $J_{\text {rad }}$ were determined by: ${ }^{26}$

$$
\begin{gathered}
J_{\mathrm{ph}}=C \times e \int_{\mathbf{E}_{\mathrm{g}}}^{\infty} \mathrm{d} \hbar \omega \frac{\Lambda}{\mathrm{d} \hbar \omega}, \\
J_{\text {rad }}=\frac{e\left(n_{\mathrm{top}}{ }^{2}+n_{\mathrm{bottom}^{2}}\right)}{4 \pi^{2} c^{2}} \int_{\mathbf{E}_{\mathrm{g}} / \hbar}^{\infty} \omega^{2} \exp \left(\frac{e V-\hbar \omega}{k T}\right) \mathrm{d} \omega,
\end{gathered}
$$




$$
J_{\mathrm{th}}=\frac{\left(n_{\mathrm{top}}{ }^{2}+n_{\mathrm{bottom}^{2}}\right)}{4 \pi^{2} c^{2}} \int_{\mathbf{E}_{\mathfrak{g}} / \hbar}^{\infty} \omega^{2} \exp \left(-\frac{\hbar \omega}{k T}\right) \mathrm{d} \omega .
$$

where $e$ is the unsigned charge on an electron, $\Lambda$ is the wavelength-dependent solar flux in the Air Mass (AM)1.5 solar spectrum, $\hbar$ is an abbreviation for $h / 2 \pi$ (with $h$ being Planck's constant), $\omega$ is the frequency of the incident light, $n_{\text {top }}$ and $n_{\text {bottom }}$ are the refractive indices of the top and the bottom of the tandem absorber, $T$ and $V$ are the operating temperature and the operating voltage, respectively, and $\mathbf{E}_{g}$ is the band gap of the top or bottom light absorber, as indicated by the appropriate subscript.

Several approaches have been proposed to effectively utilize even highly optically absorbing electrocatalysts in solar-fuels generators, including deposition of catalysts on the back side of the structure to reflect light back into the absorbers, deposition of catalysts along the surfaces or at the bases of microwire arrays to reflect light into the internal volume of the lightabsorber structure, or deposition of catalysts in pre-determined islands on planar photoelectrode structures to minimize obscuration. ${ }^{27-31}$ Any optical obscuration by the electrocatalysts would lower all of the efficiencies calculated herein by approximately the ratio of transmitted light to the light reflected outside of the specific light-absorber structure of concern. A more specific analysis of the variation of efficiency with light intensity has been performed separately for several specific system geometries. ${ }^{17}$

The numerical relationship between the current density and voltage obtained from the Shockley-Queisser model was fitted using the ideal diode relationship:

$$
J=J_{\text {th }}-J_{0}\left[\exp \left(\frac{e V}{\gamma k T}\right)-1\right]
$$

where $J_{0}$ is the reverse saturation current density and $\gamma$ is the diode ideality factor. In the sensitivity analysis, the value of $J_{\mathrm{ph}}$ was not changed for a given light absorber, because $J_{\mathrm{ph}}$ is given by the relationship between the wavelength-dependent absorption behavior of the semiconductor and the spectral irradiance of incident sunlight, as expressed by eqn (4)-(6). In contrast, the value of $J_{0}$ was increased by as much as 21 orders of magnitude from the Shockley-Queisser limit, to account for a range of materials properties of the light absorbers of interest. This variation in $J_{0}$ produced lower open-circuit voltages for a given light absorber at a given value of $J_{\mathrm{ph}}$, with the relationship between $J_{0}$ and $V_{\text {oc }}$ given explicitly by eqn (7).

\section{Behavior of electrocatalysts, membrane separator and solution electrolyte}

The current density, $J_{\mathrm{OER} / \mathrm{HER}}$ as a function of the overpotential, $\eta$, for the OER and HER can be described by the Butler-Volmer equation: ${ }^{32}$

$$
\begin{aligned}
J_{\mathrm{OER} / \mathrm{HER}} & =J_{0, \mathrm{OER} / \mathrm{HER}}\left[\exp \left(\frac{\alpha_{\mathrm{a}, \mathrm{OER} / \mathrm{HER}} F \eta}{R T}\right)\right. \\
& \left.-\exp \left(-\frac{\alpha_{\mathrm{c}, \mathrm{OER} / \mathrm{HER}} F \eta}{R T}\right)\right]
\end{aligned}
$$

where $J_{0, \mathrm{OER} / \mathrm{HER}}$ is the exchange-current density for the OER or HER, respectively, and $\alpha_{\mathrm{a}, \mathrm{OER} / \mathrm{HER}}$ and $\alpha_{\mathrm{c}, \mathrm{OER} / \mathrm{HER}}$ are the anodic and cathodic transfer coefficients for the OER or the HER, respectively. Note that use of the Butler-Volmer equation to describe the overpotential of the electrocatalysts as a function of current density produces a different overvoltage at each current density in the operational system, as opposed to assuming a fixed voltage drop for a given set of light absorbers independent of whether the system is operating at open circuit (no current passed), at the light-limited current density, at the maximum power point of the system, or with additional ohmic resistance drops due to the cell design.

The exchange-current density for the OER or the HER is dependent on temperature, and was calculated using:

$J_{0, T, \mathrm{OER} / \mathrm{HER}}=J_{0, T_{\mathrm{ref}}, \mathrm{OER} / \mathrm{HER}} \exp \left(\frac{E_{\mathrm{a}, \mathrm{OER} / \mathrm{HER}}}{R T_{\text {ref }}}\right) \exp \left(-\frac{E_{\mathrm{a}, \mathrm{OER} / \mathrm{HER}}}{R T}\right)$,

where $E_{\mathrm{a}, \mathrm{OER} / \mathrm{HER}}$ is the activation energy for the OER or HER, respectively, and $J_{0, T_{\mathrm{ree}}, \mathrm{OER} / \mathrm{HER}}$ is the exchange-current density for the OER or HER, respectively, at the reference temperature. $E_{\mathrm{a}, \mathrm{OER} / \mathrm{HER}}$ was set to $42560 \mathrm{~J} \mathrm{~mol}^{-1}$ and $28900 \mathrm{~J} \mathrm{~mol}^{-1}$ for iridium oxide and platinum catalysts, respectively, in $1 \mathrm{M}$ $\mathrm{H}_{2} \mathrm{SO}_{4}(\mathrm{aq}) .^{33}$

The behavior of each electrocatalyst and of each anodic/ cathodic electrocatalyst system was described by reference to a figure-of-merit, $\eta_{\text {overpotential }}^{10 \mathrm{~mA} \mathrm{~cm}^{-2}}$, which specified the overpotential required by that electrocatalyst (or electrocatalyst system) on an otherwise ideally nonpolarizeable electrode (or anode and cathode combination) to provide a current density of $10 \mathrm{~mA}$ $\mathrm{cm}^{-2}$. This figure-of-merit allowed for a concise description of the relevant Butler-Volmer properties of the electrocatalysts according to eqn (8) and (9).

The temperature dependence of the effective transport resistance, $R_{\text {eff }}$, was defined as:

$$
R_{\text {eff }, T}=\frac{R_{\text {eff }, \text { ref }}}{\left(1+\alpha\left[T-T_{\text {ref }}\right]\right)},
$$

where the reference temperature, $T_{\text {ref }}$, is $300 \mathrm{~K}$ and the coefficient $\alpha$ was taken to be $0.019 \mathrm{~K}^{-1}$, by fitting the experimental data for $1.0 \mathrm{M}$ sulfuric acid. ${ }^{34}$

Based on eqn (1)-(10), a zero-dimensional (0-D) analysis of the STH conversion efficiency was obtained, in which the tandem photoabsorbers, electrocatalysts, liquid electrolyte and membrane separators were coupled in series and the optical absorption, photo-carrier transport and ionic transport were coupled in parallel. The electrode surfaces were assumed to be isopotential surfaces, and the spatial inhomogeneity of the current-density distribution along the electrodes was approximated by the use of a 0-D effective transport resistance. Note that while the 0-D load-line analysis captures the key performance characteristics of an integrated photoelectrolysis system, the detailed device construct and geometrical parameters, and their influence on the current density and potential distribution in an actual three-dimensional operating system, are not elucidated in such an analysis. 
The 0-D current-voltage model could also be employed to evaluate $\eta_{\mathrm{STH}}$ for a system constructed using a discrete photovoltaic device connected in series to a discrete electrolyzer (PV + electrolyzer), in which the efficiencies of the photovoltaic device and of the electrolyzer were optimized independently. A comparison between the dependence on temperature and illumination intensity dependence of $\eta_{\mathrm{STH}}$ for an integrated photoelectrochemical system and for a stand-alone PV + electrolyzer system has been described previously. ${ }^{17}$ The low resistive loss due to a lower operating current density in the integrated system, or "distributed internal electrolyzer", especially at low illumination intensities, and the enhanced kinetics and transport at elevated temperatures in the integrated designs are the main reasons that an integrated system outperformed a standalone PV + electrolyzer system under the conditions evaluated in that work.

\section{Results and discussion}

\section{State-of-the-art component properties}

(a) Electrocatalysts. The polarization behavior for the overall water-splitting process is dependent on the combined interfacial kinetics of the HER and the OER catalysts. ${ }^{32}$ A reduction in the overpotential for the HER and/or for the OER has been one of the major research goals of electrocatalyst development. ${ }^{21}$ Some of the most active electrocatalysts reported to date operate in aqueous alkaline media, including Ni-Mo alloys for the HER (overpotential of $75 \mathrm{mV}$ at $10 \mathrm{~mA} \mathrm{~cm}^{-2}$ with a Tafel slope of $40 \mathrm{mV} \mathrm{dec}{ }^{-1}$ ) and (Fe-Ni) $\mathrm{O}_{x}$ alloys for the OER (overpotential of $280 \mathrm{mV}$ at $10 \mathrm{~mA} \mathrm{~cm}^{-2}$ with a Tafel slope of 40 $\left.\mathrm{mV} \mathrm{dec}^{-1}\right){ }^{35,36}$ In acidic aqueous solutions, the state-of-the-art electrocatalysts for HER and OER contain noble metals and metal oxides, such as Pt and $\mathrm{IrO}_{x}$, which operate at $55 \mathrm{mV}$ and $270 \mathrm{mV}$ overpotential at $10 \mathrm{~mA} \mathrm{~cm}{ }^{-2}$ for the HER and OER, respectively, ${ }^{5,37}$ although recent work with transition-metal phosphides has shown overpotentials for the HER that approach that of $\mathrm{Pt}^{38-40}$ Recent benchmarking work ${ }^{\mathbf{2 1}}$ has shown that in aqueous alkaline solutions, many active nonnoble metal electrocatalysts for the OER exhibit mutually similar overpotentials, of between $350 \mathrm{mV}$ and $430 \mathrm{mV}$, at an operating current density of $10 \mathrm{~mA} \mathrm{~cm} \mathrm{~cm}^{-2}$. In addition, under acidic conditions, no reported active OER electrocatalyst is stable under anodic operational conditions except for $\operatorname{IrO}_{x}{ }^{21}$

The total kinetic overpotential, $\eta_{\text {overpotential }}^{10 \mathrm{~mA} \mathrm{~cm}^{-2}}$, used to characterize the behavior of the electrocatalysts was the sum of the overpotentials for the OER and HER at $10 \mathrm{~mA} \mathrm{~cm}^{-2}$, and was dependent on the exchange-current density and the Tafel slopes for the OER and HER, all of which were varied systematically in this analysis. For example, the current-voltage relationship for the HER was fixed, and the exchange-current density for the OER, $J_{0 \text {,OER }}$, was varied from $1.1 \times 10^{-2} \mathrm{~mA} \mathrm{~cm}^{-2}$ to $1.1 \times 10^{-46} \mathrm{~mA} \mathrm{~cm}^{-2}$. This procedure resulted in total electrocatalyst overpotentials ranging from $194 \mathrm{mV}$ to $1965 \mathrm{mV}$ at the current density of $10 \mathrm{~mA} \mathrm{~cm}^{-2}$. The total overpotential at 10 $\mathrm{mA} \mathrm{cm}{ }^{-2}$ of current density was a concise, useful figure-of-merit used herein to differentiate between, and identify, the various different electrocatalyst combinations, but the actual operating current densities of each tandem absorber/electrocatalyst combination in the operating system of interest were calculated individually for each system using the load-line analysis of eqn (1). Because the total overpotential is the important system-level quantity, the procedure used herein to designate and vary the behavior of the electrocatalysts was general for variation in either the exchange-current density of the OER or the HER, or both.

(b) Light absorbers. For light absorbers, a tandem structure can produce significantly higher solar energy-conversion efficiencies than a single-junction system. The optimal solar-tohydrogen conversion efficiency is highly dependent on the combination of band gaps of the tandem light absorbers. ${ }^{\mathbf{1 4 , 1 9}}$ For instance, under 1 Sun illumination with an Air Mass (AM) 1.5 solar spectrum, the optimal top/bottom semiconductor band-gap combination is $1.65 \mathrm{eV} / 0.95 \mathrm{eV}$, which could yield, at the detailed-balance limit, a solar-to-hydrogen conversion efficiency of $31.1 \%$ in a system using $\mathrm{Pt}$ and $\mathrm{IrO}_{x}$ electrocatalysts and an optimized system design that minimizes the solution resistance $\left(0.1 \mathrm{ohm} \mathrm{cm}^{-2}\right) .{ }^{19}$

However, the discovery of stable and high-performing lightabsorber materials that are comprised of earth-abundant elements and that have a band gap of $\sim 1.6-1.8 \mathrm{eV}$ has proven challenging. The reported energy-conversion efficiency and current-voltage performance of state-of-the-art light-absorber materials for the top cell, i.e., $\mathrm{BiVO}_{4}, \mathrm{FeO}_{x}$, and $\mathrm{WO}_{3}$, are far below the $\mathrm{S}-\mathrm{Q}$ limit. Additionally, the band gaps of these materials are far from the ideal band gap for the top cell of a system. For instance, state-of-the art $\mathrm{WO}_{3}$ prepared by electrodeposition or sputtering exhibits a solar energy-conversion efficiency of $\leq 1.3 \%$ in contact with $1 \mathrm{M} \mathrm{H}_{2} \mathrm{SO}_{4}(\mathrm{aq}){ }^{41,42}$ The misalignment between the conduction band of the $\mathrm{WO}_{3}$ and the Nernstian potential for the OER, as well as charge-carrier recombination at the surface and in the bulk, result in an opencircuit voltage, $650 \mathrm{mV}$, that is low considering the large band gap of $\mathrm{WO}_{3}(2.6-2.7 \mathrm{eV}){ }^{41,42}$

(c) System design space. The detailed geometry of the cell construct, as well as the choice of the solution electrolyte, also can have a significant impact on the overall solar-to-hydrogen system efficiency. ${ }^{18}$ Targeted geometric parameters for various types of cells, including a vapor-feed solar-driven water-splitting system and a $10 \times$ solar concentrator-assisted water-splitting system, have been explored in detail. ${ }^{\mathbf{1 6 , 2 0}}$ The resistive loss, concentration overpotential and effects of electrodialysis of different electrolytes, including strong base/acid and buffered solutions has also been investigated. ${ }^{43}$ When an optimal cell configuration and strongly acidic or alkaline electrolytes are employed, the average resistive loss in the membrane separator and solution electrolyte can be limited to less than $100 \mathrm{mV}$ at an operational current density of $10 \mathrm{~mA} \mathrm{~cm}{ }^{-2} \cdot{ }^{17,18}$ In this work, the effective transport resistance was set to $10 \mathrm{ohm} \mathrm{cm}^{-2}$, which resulted in $100 \mathrm{mV}$ potential loss at $10 \mathrm{~mA} \mathrm{~cm}^{-2}$. These resistive losses were accounted for in the calculation of $\eta_{\mathrm{STH}}$ in this study, along with the kinetic overpotential losses due to the water-splitting reaction. The voltage losses were not fixed relative to the band gap regardless of the actual system operating conditions, ${ }^{\mathbf{1 4 , 4 4}}$ but instead, as given by eqn (1)-(10), the voltage 
losses were explicitly a function of the operating voltage and current, in conjunction with the current-dependent ohmicbased voltage losses and kinetically based electrocatalyst overpotential losses, in the specific system being evaluated.

The sensitivity analysis investigated herein is applicable to several specific device designs, including a "closed-sandwich" design in which the photocathode and photoanode are assembled back-to-back, ${ }^{18}$ an "open-sandwich" design with suitable spectral-splitting in which the photocathode and photoanode are assembled side-by-side, ${ }^{18}$ a one-dimensional "trough" design and two-dimensional "bubble-wrap" design for the photoelectrochemical cells coupled to solar concentrators, ${ }^{16}$ and a membrane-enclosed, vapor-feed design. ${ }^{20}$ The analysis is not applicable to device designs in which the geometric area of the electrolyzing components is significantly different from that of the light absorbers.

(d) Membranes/separators. Depending on the specific device designs, the permeability and conductivity of the membrane separator could impact the overall $\eta_{\mathrm{STH}}$ of the device. Ion transport through the separator is necessary and results in a resistive loss in the system. Nevertheless, many highly conductive polymers, e.g., Nafion $\left(10 \mathrm{~S} \mathrm{~m}^{-1}\right)$, exhibit minimal resistive loss at $10 \mathrm{~mA} \mathrm{~cm} \mathrm{~cm}^{-2}$ in optimized device geometries. ${ }^{17,18}$ Another primary function of a membrane separator is to block the diffusive and convective crossovers for the product gases. In certain microstructured cell designs, $\eta_{\mathrm{STH}}$ is highly dependent on the permeability of the membrane materials. ${ }^{45}$ Through use of a thick membrane separator $(50 \mu \mathrm{m}$ to $100 \mu \mathrm{m}$ ) and/or with an optimized cell geometry, the efficiency loss due to product crossover can be negligible. ${ }^{17,18,45}$

\section{Optimal STH conversion efficiency at different total overpotentials}

In the "one factor at a time" sensitivity analysis performed herein, the performance that can be obtained for various families of tandem light absorbers has been investigated in detail, with the families of tandem light absorbers designated either by operation at the $\mathrm{S}-\mathrm{Q}$ limit or by containing various levels of defects that will degrade the photovoltage by increasing the $J_{0}$ of the absorbers away from the $\mathrm{S}-\mathrm{Q}$ limit. In this process, for a given set of electrocatalysts (denoted by their total overpotential at $10 \mathrm{~mA} \mathrm{~cm} \mathrm{~cm}^{-2}$ of current density, $\eta_{\text {overpotential }}^{10 \mathrm{~mA}}$, as a concise figure-of-merit descriptor, but not constrained to operate at $10 \mathrm{~mA} \mathrm{~cm}{ }^{-2}$ in the actual system of interest), the band gaps of the tandem light absorbers were varied to ascertain the optimally performing tandem light-absorber combination for a given set of OER and HER electrocatalysts.

Hence, for a given set of electrocatalysts, the analysis identified the maximum system efficiency, $\eta_{\mathrm{STH}, \mathrm{opt}}$, that could be obtained through use of the set of light absorbers that were identified as optimal with those specific electrocatalysts. In addition, for that same specific set of electrocatalysts, the optimization process was repeated, and a separate tandemabsorber combination was identified to give the optimum system efficiency, but with the light absorbers having their photovoltage degraded by a specified amount from the $\mathrm{S}-\mathrm{Q}$ limit, as described by an increased exchange-current density (and consequently decreased photopotential at constant charge-carrier injection level) for this separate set of tandem light absorbers. Thus, a different set of tandem light absorbers produced optimal system efficiencies for each specified combination of electrocatalysts and for each family of light absorbers (as quantified by the increase in $J_{0}$ of the light absorbers relative to the $\mathrm{S}-\mathrm{Q}$ limit).

Note that this process produces a fundamentally different outcome, and answers a fundamentally different question, than evaluating the degradation in efficiency of a specific set of tandem light absorbers as a function of increases in the overpotentials of the electrocatalysts. In the latter approach, the optimum band-gap combination for a tandem structure is identified in the absence of any system losses, and then the decrease in efficiency is evaluated in response to increases in assumed voltage losses in the system. ${ }^{\mathbf{1 4 , 4 4}}$ In such a situation, modest increases in the electrocatalyst overpotential can produce large decreases in the resulting system efficiency, especially when the tandem structure is designed to barely provide sufficient photovoltage to drive the electrocatalysts at near the light-limited current density during operation at optimal performance. In practice, such higher overpotential catalysts would instead optimally be utilized in conjunction with light absorbers that themselves had higher band gaps, to yield higher system efficiencies by allowing for operation at or near the light-limited current density with the specific set of electrocatalysts of interest. The optimized efficiency, $\eta_{\mathrm{STH}, \mathrm{opt}}$, of the latter system would be lower than that of the former system, due to the a slight decrease in the light-limited current density arising from the required increase in band gaps of the newly optimized tandem light absorbers, but the optimized efficiency would not be nearly as low as the efficiency of a system in which the tandem-absorber band gaps were fixed in the design and implementation phases and thus did not drive the electrocatalysts being used in that system at the highest possible current densities during system operation.

The process used herein is specifically illustrated in Fig. 1ad. Using the electrocatalytic performance of the state-of-the-art electrocatalysts as described by the $\mathrm{B}-\mathrm{V}$ relation (for an electrocatalyst system described concisely by the figure-of-merit having a value of $\eta_{\text {overpotential }}^{10 \mathrm{~mA} \mathrm{~cm}-2}=355 \mathrm{mV}$ ), the band-gap combination of the tandem photoabsorbers was optimized and yielded $\eta_{\mathrm{STH} \text {,opt }}$ at the $\mathrm{S}-\mathrm{Q}$ limit for absorbers having a tandem band-gap combination of $1.7 \mathrm{eV} / 1.0 \mathrm{eV}$. In this "photocathode + photoanode" optimization process, for each combination of light absorbers and electrocatalysts, a load-line analysis based on two half-cell current-voltage characteristics was used, and the intersection point described the operating current of the full cell derived from these two half-cells. The green curves in Fig. 1a represent the resulting current-voltage relation of the photocathode and the photoanode, in which the operating current density produced $\eta_{\mathrm{STH}, \mathrm{opt}}=\eta_{\mathrm{STH}, \mathrm{a}}=27.5 \%$.

The electrocatalytic performance of the HER catalyst was then fixed, and the overpotential of the OER catalyst was increased by decreasing the exchange-current density 
a.

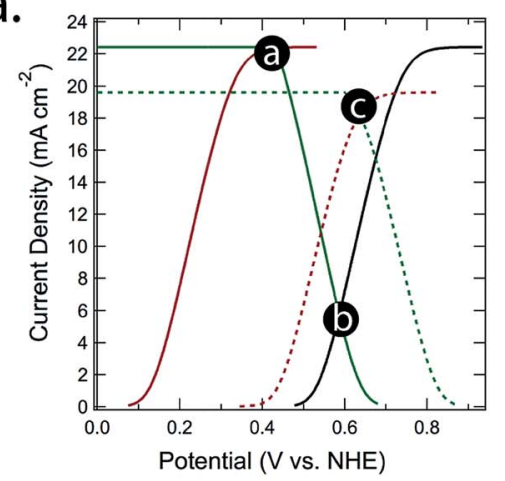

C.

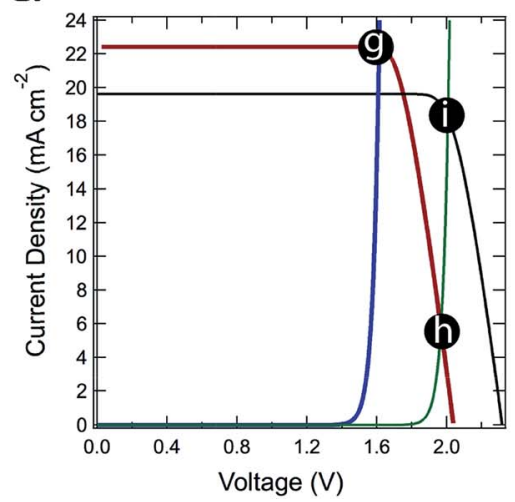

b.

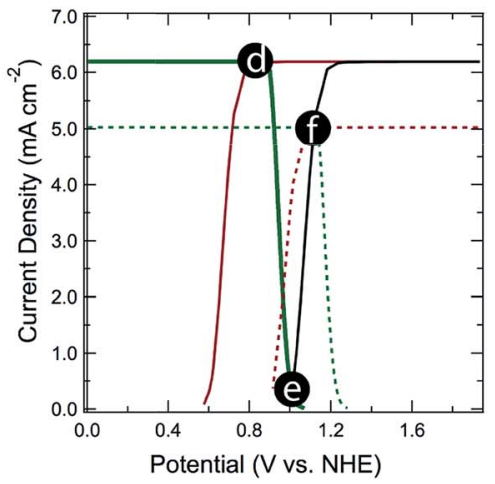

d.

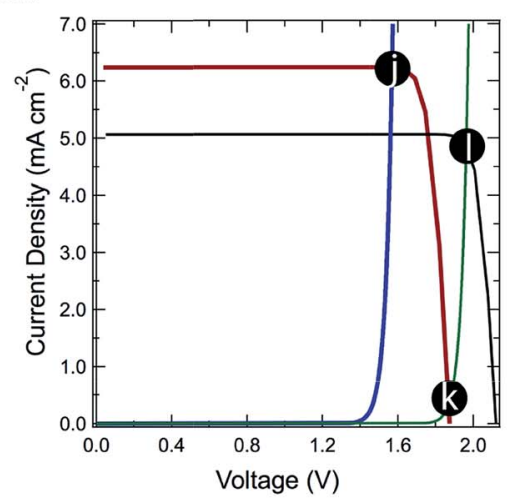

Fig. 1 Analysis of the operating current density of solar-driven water-splitting cells using a photocathode + photoanode analysis (a and b) and a tandem photoabsorber + overall loading curve analysis (c and d). The photoabsorbers performed at the S-Q limit in (a) and (c) and performed at the reverse-saturation current density of $10^{21} \mathrm{~J}_{0}$ in (b) and (d).

$\left(\eta_{\text {overpotential }}^{10 \mathrm{~mA} \mathrm{~cm}^{-2}}=757 \mathrm{mV}\right)$. As shown in black in Fig. 1a, the new operating point that resulted from this increase in catalyst overpotential produced a very large decrease in system efficiency, to $\eta_{\mathrm{STH}, \mathrm{b}}=7.1 \%$. However, for this new set of electrocatalysts, the original tandem light-absorber structure no longer provides the band-gap combination that provides the highest possible system efficiency. When the band gaps of the tandem system were reoptimized in response to the increased overpotential of this new electrocatalyst system, with the constraint that the absorbers were still performing at the S-Q limit, the half-cell behavior in Fig. 1a in blue was obtained, and the resultant operating point yielded $\eta_{\mathrm{STH}, \mathrm{opt}}=\eta_{\mathrm{STH}, \mathrm{c}}=22.4 \%$. The light-limited current density of these new tandem absorbers is lower than the light-limited current density of the absorbers that were used to obtain the curves in green (and yield operating points a or b) in Fig. 1, because the band gaps of the new optimally performing system were increased (and thus $J_{\mathrm{ph}}$ decreased for a given illumination intensity and spectral distribution from the sun) relative to the band gaps of the original system (in a and b). However, the system efficiency with the specified electrocatalysts is much higher for the newly optimized system, because the increased band gaps provide a sufficient increase in photovoltage to overcome the increased catalyst overpotentials, and therefore allow system operation at point c, near the light-limited current density of the newly optimized tandem light absorber combination, with band gaps of $1.8 \mathrm{eV} / 1.2 \mathrm{eV}$. Reoptimization of the band gaps of the tandem structure therefore allowed the design of a system with a much higher system efficiency for these degraded electrocatalysts than the system efficiency obtained by fixing the properties of the tandem structure based on negligible system losses, and then absorbing the entirety of any real system voltage losses directly in the form of decreases to the resulting system efficiency.

In a subsequent step, the overall optimum system efficiency $\eta_{\text {STH,opt }}$ was recalculated for each specific electrocatalyst system of interest, but with the constraint that the light absorbers were degraded in performance from the S-Q limit by a specified increase in $J_{0}$. For example, for the original electrocatalyst behavior corresponding to the blue half-cell curves in Fig. 1a, the green curves in Fig. 1b described the half-cell characteristics of the tandem structure in which $J_{0}$ was increased by $10^{21}$ relative to the values of $J_{0}$ obtained from the $\mathrm{S}-\mathrm{Q}$ limit. For this specific combination of electrocatalysts, the optimal system efficiency was very low, corresponding to $\eta_{\mathrm{STH}, \mathrm{opt}}=\eta_{\mathrm{STH}, \mathrm{d}}=$ $7.7 \%$. Due to the low photovoltage of the degraded light absorbers relative to the values of their band gaps, the optimal system efficiency for this set of electrocatalyst properties required a very significant increase in the band gap of the absorbers, to values of $2.5 \mathrm{eV} / 1.9 \mathrm{eV}$, to drive the electrocatalysts and thus produced low overall optimal system efficiencies. For these tandem light-absorber systems, the same increase in 
electrocatalyst overpotential as that represented in Fig. 2a produced operating point e in Fig. 2b, with a corresponding decrease in efficiency to $\eta_{\mathrm{STH}, \mathrm{e}}=0.4 \%$. Reoptimization of the band gaps, however, produced a system having significantly higher efficiencies, as represented by the intersection of the red and the green curves in Fig. $1 \mathrm{~b}$ at operating point $\mathrm{f}\left(\eta_{\mathrm{STH}, \mathrm{op}}=\right.$ $\left.\eta_{\mathrm{STH}, \mathrm{f}}=6.0 \%\right)$.

A different approach, the "tandem photoabsorber + overall loading curve" configuration (Fig. 1c and d), was also employed to obtain the $\eta_{\mathrm{STH}}$ of the system, which yielded identical results to the half-cell load-line analysis depicted in Fig. 1a and b. In Fig. $1 \mathrm{c}$ and $\mathrm{d}$, the blue and the green curves represent the overall water-splitting loading curves with $\eta_{\text {overpotential }}^{10 \mathrm{~mA} \mathrm{~cm}^{-2}}=355 \mathrm{mV}$ and $\eta_{\text {overpotential }}^{10 \mathrm{~mA} \mathrm{~cm}-2}=757 \mathrm{mV}$, respectively. The red curve represents the current-voltage characteristic of the tandem photoabsorbers, in which the band-gap combination was optimized $(1.7 \mathrm{eV} / 1.0 \mathrm{eV})$ for the blue loading curve. The resulting $\eta_{\mathrm{STH}, \mathrm{opt}}=\eta_{\mathrm{STH}, \mathrm{g}}$ was $27.5 \%$, which corresponds to the operating current density at point $\mathrm{g}$. The $\eta_{\mathrm{STH}, \mathrm{h}}$ decreased to $7.1 \%$, which corresponds to the operating point $\mathrm{h}$, when the activity of the electrocatalysts decreased (blue loading curve) without the reoptimization of the band-gap combination. When the band-gap combination was optimized $(1.8 \mathrm{eV} / 1.2 \mathrm{eV})$ for the green loading curve, the resulting $\eta_{\mathrm{STH}, \mathrm{opt}}=\eta_{\mathrm{STH}, \mathrm{i}}$ was increased to $22.4 \%$, which corresponds to the operating point i. The same trend was observed in Fig. 2d, in which the quality of the tandem photoabsorber was varied by changing the reverse-saturation current density to $10^{21} J_{0}\left(\eta_{\mathrm{STH}, \mathrm{j}}=7.7 \%, \eta_{\mathrm{STH}, \mathrm{k}}=0.4 \%\right.$ and $\eta_{\mathrm{STH}, \mathrm{l}}=$ $6.0 \%)$.

Fig. 2a plots $\eta_{\mathrm{STH}, \mathrm{opt}}$ for the system design space of interest, incorporating the optimized tandem-cell arrangement, as a function of the behavior of various different combinations of electrocatalysts. As described above, at each value of the total overpotential, the entire suite of band-gap combinations for the tandem cell was explored to identify the combination that produced the optimum efficiency, $\eta_{\mathrm{STH}, \mathrm{opt}}$, at the specified total system overpotential. In this process, the band gap of the top light absorber was varied from $1.3 \mathrm{eV}$ to $3.0 \mathrm{eV}$, and band gap of the bottom light absorber ranged between $0.6 \mathrm{eV}$ and $2.0 \mathrm{eV}$.

The numerical current-voltage characteristic of the light absorbers in the tandem cell under the Shockley-Queisser (S-Q) limit (blue curve) was obtained using the ideal photodiode relationship. As shown in the top-most data set plotted in Fig. 2a, for light absorbers operating at the detailed-balance limit, the slope of $\eta_{\mathrm{STH} \text {,opt }}$ as a function of the total overpotential, $\frac{\Delta \eta_{\mathrm{STH}, \mathrm{opt}}}{\Delta \eta_{\text {overpotential }}^{10 \mathrm{~mA} \mathrm{~cm}-2}}$, was $\sim 0.01 \% \mathrm{mV}^{-1}$. Hence, decreases in the total overpotential by $100 \mathrm{mV}$, from $400 \mathrm{mV}$ to $300 \mathrm{mV}$, would result in 1 percentage-point change in $\eta_{\mathrm{STH} \text {,opt }}$. State-ofthe-art electrocatalysts in alkaline solution, such as Ni-Mo alloy

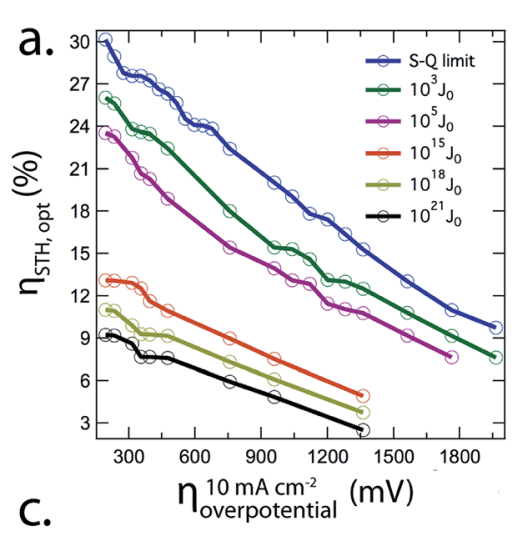

b.
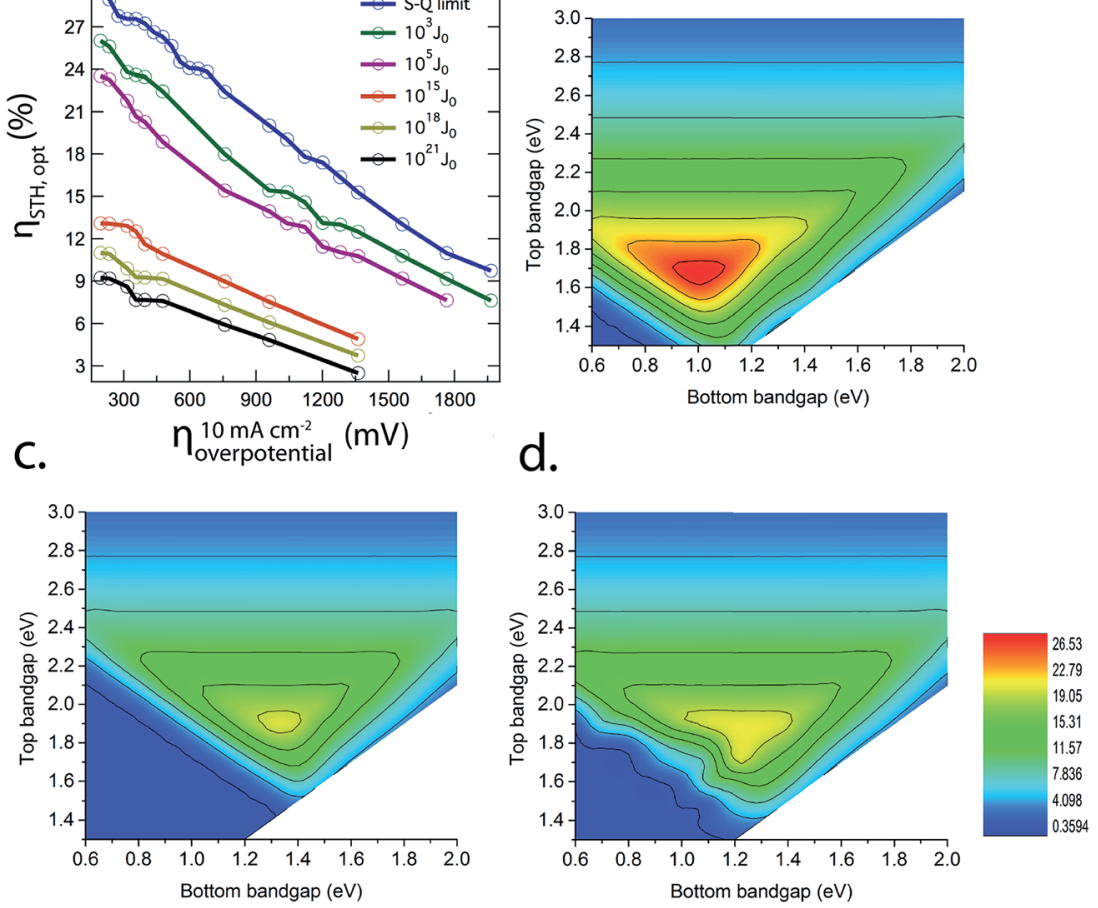

Fig. 2 (a) Optimal STH conversion efficiency, $\eta_{\mathrm{STH}, \mathrm{opt}}$ at all band-gap combinations as a function of the electrocatalyst characteristics described by the total electrocatalytic overpotential at $10 \mathrm{~mA} \mathrm{~cm}{ }^{-2}$ for the hydrogen-evolution reaction (HER) and the oxygen-evolution reaction (OER). The actual operating current densities for each system were obtained using a load-line analysis, as described in Fig. 1 . The reverse-saturation current densities for the photoabsorbers were swept from the Shockley-Queisser (S-Q) limit, $J_{0}$, to $10^{21} J_{0}$. (b) $\eta_{S T H, \text { opt }}$ as a function of the top and bottom band-gap combinations when the reverse-saturation current density and the total overpotential at $10 \mathrm{~mA} \mathrm{~cm}^{-2}$ were set to $\mathrm{J}_{0}$ and $355 \mathrm{mV}$ and (c) $\mathrm{J}_{0}$ and $959 \mathrm{mV}$ and (d) $10^{5} \mathrm{~J}_{0}$ and $355 \mathrm{mV}$, respectively. 
for the HER and $\mathrm{NiFeO}_{x}$ mixed-metal oxides for the OER, exhibit $75 \mathrm{mV}$ and $280 \mathrm{mV}$ overpotential, respectively, at operating current densities of $10 \mathrm{~mA} \mathrm{~cm} \mathrm{~cm}^{-2}$. The calculation that corresponds to this particular catalyst system at the detailed-balance limit is shown in Fig. $2 \mathrm{~b}$, where $\eta_{\mathrm{STH} \text {,opt }}$ is $27.5 \%$ at the band-gap combination of $1.7 \mathrm{eV} / 1.0 \mathrm{eV}$. Improving the existing catalyst system by decreasing the overpotential for the OER by $100 \mathrm{mV}$ at a current density of $10 \mathrm{~mA} \mathrm{~cm} \mathrm{~cm}^{-2}$, which would represent very significant progress in electrocatalyst development, would result in a potential possible efficiency improvement of 1 percentage point in $\eta_{\mathrm{STH} \text {,opt }}$ for the full system.

The reverse-saturation current density of the ideal photodiode at the detailed-balance limit, $J_{0}$, was then increased from $J_{0}$ to $10^{21} J_{0}$, while the light-limited photocurrent density was maintained constant. This procedure was used to account for a very substantial degradation in the properties of the light absorbers relative to operation at the theoretical detailedbalance limit. The resulting open-circuit voltages were decreased significantly due to the increase of the reverse-saturation current densities. In fact, during this process, $J_{0}$ was increased to a value such that overall system efficiencies could not exceed 5\% even with essentially zero overpotential and solution-resistance losses combined. For all values of $J_{0}$, the slope of $\eta_{\mathrm{STH}, \mathrm{opt}}$ as a function of the total overpotential, $\frac{\Delta \eta_{\mathrm{STH}, \mathrm{opt}}}{\Delta \eta_{\text {overpotential }}^{10 \mathrm{~mA} \mathrm{c}-2}}$, was approximately $0.01 \% \mathrm{mV}^{-1}$.

As the total overpotential increased or as the reverse-saturation current density increased, the light-absorber combination that produced $\eta_{\mathrm{STH} \text {,opt }}$ had higher band-gap values than the base case. Fig. $2 \mathrm{c}$ and d show $\eta_{\mathrm{STH} \text {,opt }}$ for all band-gap combinations when the total overpotential was $959 \mathrm{mV}$ at the detailedbalance limit and the total overpotential was $355 \mathrm{mV}$ with the reverse-saturation current density set to $10^{5} J_{0}$, respectively. The corresponding optimal band-gap combination was $2.0 \mathrm{eV} / 1.2 \mathrm{eV}$ in Fig. 2c and was $1.9 \mathrm{eV} / 1.2 \mathrm{eV}$ in Fig. 2d.

Although the state-of-the-art electrocatalysts in alkaline solution exhibit a total overpotential of $\sim 350 \mathrm{mV}$ at $10 \mathrm{~mA}$ $\mathrm{cm}^{-2}$, in acidic conditions, a paucity of stable earth-abundant electrocatalysts for the OER have been reported, and thus development of an earth-abundant OER catalyst that is stable in acid would be a significant improvement upon the state-of-theart. ${ }^{21}$ Moreover, when the cathodic reaction involves the $\mathrm{CO}_{2}$ reduction reaction, large overpotentials, typically over $1 \mathrm{~V}$, are observed. ${ }^{46}$ In these less-developed materials systems, improvements in the catalytic performance would result in significant enhancement of $\eta_{\mathrm{STH}, \mathrm{opt}}$.

Note that while the general trend,

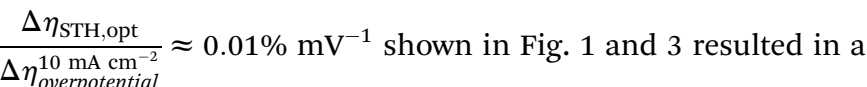
marginal change of $\eta_{\mathrm{STH}, \mathrm{opt}}$ in response to the catalyst performance, significant change in the relative STH conversion efficiency could occur in low efficiency cells. For instance, when the OER overpotential at $10 \mathrm{~mA} \mathrm{~cm}{ }^{-2}$ was reduced from $400 \mathrm{mV}$ to $200 \mathrm{mV}$, which would represent significant advancement in catalyst development, in a cell employing tandem photoabsorbers that operate close to S-Q limit, the $\eta_{\mathrm{STH}, \mathrm{opt}}$ would increase from $\sim 27.5-29.5 \%$ (by a factor of $<10 \%$ relative). Alternatively, the same decrease in OER overpotential would make a much larger relative difference in the optimal system efficiency for poorly performing, degraded light absorbers, and could for example increase $\eta_{\mathrm{STH} \text {,opt }}$ by a factor of 1.5 (i.e. by $50 \%$ relative), from $\eta_{\mathrm{STH}, \mathrm{opt}}=2 \%$ to $\eta_{\mathrm{STH} \text {,opt }}=3 \%$. However, clearly one cannot convert an inefficient optimal system design into an efficient optimized system design by decreasing the OER overpotential by $100 \mathrm{mV}$ from state-of-the-art values at the present time. Hence the sensitivity analysis clearly indicates that improvements in the light absorbers are both necessary and sufficient to enable highly increased efficiencies for optimized solar-driven water-splitting systems.

\section{General trend of the sensitivity behavior}

For electrocatalysts that follow the Butler-Volmer (B-V) relationship, the kinetic overpotential for the HER as well as for the OER not only depends on the exchange-current density but also on the transfer coefficient. ${ }^{32}$ Fig. 3a shows $\eta_{\mathrm{STH} \text {,opt }}$ at the detailed-balance limit as a function of the total overpotential at $10 \mathrm{~mA} \mathrm{~cm}{ }^{-2}$, in which the transfer coefficient for HER and OER, or in which the exchange-current density for the HER and OER, were parametrically varied. The transfer coefficients for the HER and OER were varied so that the Tafel slopes for the corre-

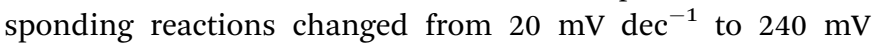
$\mathrm{dec}^{-1}$. The exchange-current density for the HER was varied from $1.4 \mathrm{~mA} \mathrm{~cm}^{-2}$ to $1.4 \times 10^{-36} \mathrm{~mA} \mathrm{~cm}{ }^{-2}$ and the exchangecurrent density for the OER was varied from $1.1 \times 10^{-2} \mathrm{~mA}$ $\mathrm{cm}^{-2}$ to $1.1 \times 10^{-41} \mathrm{~mA} \mathrm{~cm}{ }^{-2}$. At all band-gap combinations, $\eta_{\mathrm{STH} \text {,opt }}$ exhibited little dependence on the four specific parameter variations, and $\eta_{\mathrm{STH}, \mathrm{opt}}$ exhibited a quasi-linear relationship with the total overpotential at $10 \mathrm{~mA} \mathrm{~cm} \mathrm{~cm}^{-2}$. Thus, the total overpotential at a current density of $10 \mathrm{~mA} \mathrm{~cm} \mathrm{~cm}^{-2}$ is a globally useful figure-of-merit for evaluating the efficiency of the full water-splitting system in these generic system designs.

The three-dimensional transport-resistance distribution is highly dependent on the detailed design and geometric parameters of the cell as well as the ion-transport properties of the solution electrolyte. In this work, a 0-dimensional approximation was used to obtain an effective transport resistance. Fig. $3 \mathrm{~b}$ shows $\eta_{\mathrm{STH} \text {,opt }}$ as a function of the total overpotential at $10 \mathrm{~mA} \mathrm{~cm}^{-2}$ when the effective transport resistance was set to 1 ohm cm $\mathrm{cm}^{-2}, 10 \mathrm{ohm} \mathrm{cm} \mathrm{cm}^{-2}$ or $50 \mathrm{ohm} \mathrm{cm}^{-2}$, respectively. Although $\eta_{\mathrm{STH} \text {,opt }}$ showed a strong dependence on the detailed geometric parameters of the device, the slope, $\frac{\Delta \eta_{\mathrm{STH}, \mathrm{opt}}}{\Delta \eta_{\text {overpotential }}^{10 \mathrm{~mA} \mathrm{~cm}-2}}$, exhibited little dependence on the effective transport resistance. The red curve represented the optimal STH conversion efficiency with a smaller effective transport resistance. As a result, the overall efficiency was higher than the values displayed in Fig. 2 but in no case did the value exceed the S-Q limit.

\section{Operational temperature and illumination conditions}

Fig. $4 \mathrm{a}$ and b depict the sensitivity of $\eta_{\mathrm{STH} \text {,opt }}$ as a function of the operational temperature and the incident illumination concentration, respectively. The value of $\eta_{\mathrm{STH} \text {,opt }}$ exhibited a 
a.

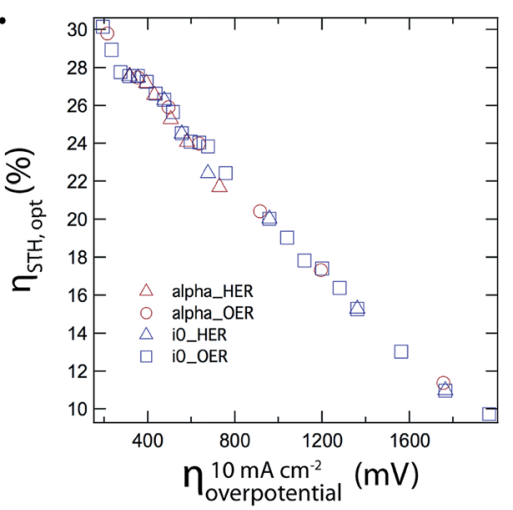

b.

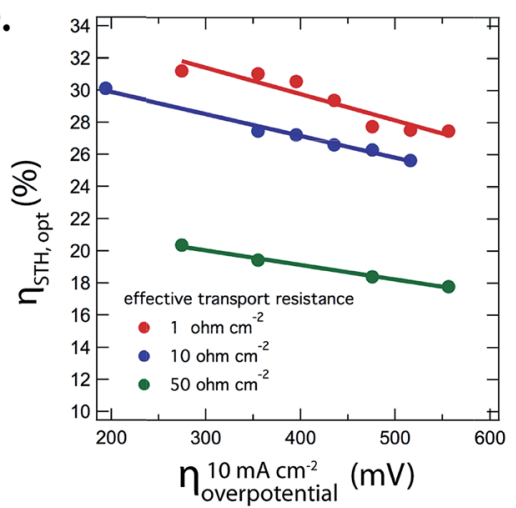

Fig. 3 (a) $\eta_{\mathrm{STH}, \mathrm{opt}}$ at all band-gap combinations as a function of the total electrocatalyst overpotential at $10 \mathrm{~mA} \mathrm{~cm}{ }^{-2}$ when the exchangecurrent density for the HER or OER, or the transfer coefficient for the HER or OER, was parametrically varied. The actual operating current densities for each system were obtained using a load-line analysis, as described in Fig. 1. (b) $\eta_{\mathrm{STH} \text {,opt }}$ at all band-gap combinations as a function of the total electrocatalyst overpotential at $10 \mathrm{~mA} \mathrm{~cm}^{-2}$ (but with the actual operating current densities for each system obtained using a load-line analysis, as described in Fig. 1) when the effective transport resistance in the solution electrolyte and membrane separators was set to 1 ohm

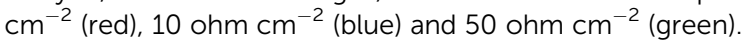

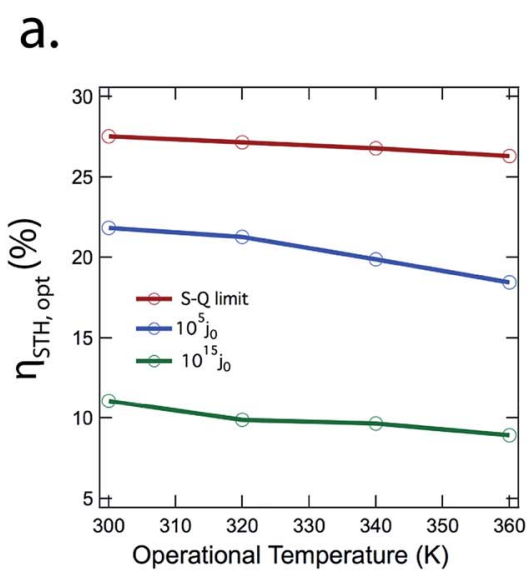

b.

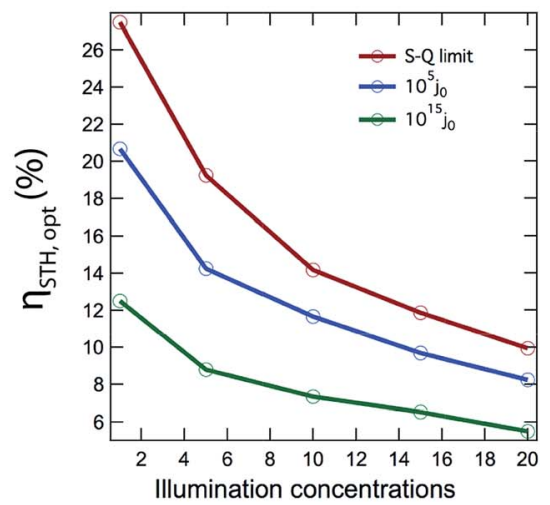

Fig. 4 (a) $\eta_{\mathrm{STH} \text {,opt }}$ at all band-gap combinations as a function of the operational temperature for the integrated system, (b) $\eta_{\mathrm{STH}, \mathrm{opt}}$ at all band-gap combinations as a function of the illumination-concentration factor for the integrated system. Three photoabsorbers having reverse-saturation current densities either at the $\mathrm{S}-\mathrm{Q}$ limit (red) $\left(\mathrm{J}_{0}\right), 10^{5} \mathrm{~J}$ (blue) or $10^{15} \mathrm{~J}$ (green) were calculated.

weak dependence on the operational temperature in the range of $T=300 \mathrm{~K}$ to $360 \mathrm{~K}$. At the detailed-balance limit, $\eta_{\mathrm{STH}, \mathrm{opt}}$ decreased from $27.5 \%$ to $26.3 \%$ when the operating temperature increased from $300 \mathrm{~K}$ to $360 \mathrm{~K}$. The weak temperature dependence resulted from two competing effects: degradation of the performance of the light absorbers and enhancement of the electrocatalytic and ion-transport rates at elevated temperatures. As the operational temperature increased, the radiative recombination in the light absorbers increased according to eqn (5), which resulted in a decrease in the performance of the light absorbers. However, as the operational temperature increased, the total overpotential for electrocatalysis and the transport resistance according to eqn (9) and (10) also decreased.

The value of $\eta_{\mathrm{STH}, \mathrm{opt}}$ exhibited a significant dependence on the optical concentration factor of the incident solar illumination. As the optical concentration increased, the resulting higher operational current density resulted in an increase in the kinetic overpotentials needed to drive the HER and OER at the required interfacial flux. As a result, $\eta_{\mathrm{STH}, \mathrm{opt}}$ at the detailedbalance limit decreased from $30.1 \%$ to $25.1 \%$ when the optical concentration increased from 1 Sun $\left(100 \mathrm{~mW} \mathrm{~cm}^{-2}\right)$ to 20 Sun $\left(2000 \mathrm{~mW} \mathrm{~cm}^{-2}\right)$.

\section{Conclusions}

The sensitivity analysis yielded a general trend for the behavior of $\eta_{\mathrm{STH}, \mathrm{opt}}$ as a function of the total overpotential for the HER and OER at $10 \mathrm{~mA} \mathrm{~cm}{ }^{-2}$, regardless of the quality of the light absorbers. In response to a change in catalyst properties, the band-gap combinations of the tandem photoabsorber were varied to then achieve the optimal STH conversion efficiency at the specified total overpotential. As the total overpotential increased, the optimal band-gap combinations shifted to higher 
band-gap values to accommodate the additional voltage requirements. Hence, a relatively small decrease in the optimal STH efficiency was observed, and a reduction in the overpotential at $10 \mathrm{~mA} \mathrm{~cm} \mathrm{~cm}^{-2}$ by $100 \mathrm{mV}$ from values for present state-of-the-art electrocatalysts resulted in $\sim 1$ percentage-point improvement of the optimally attainable STH conversion efficiency. The sensitivity factor, $\frac{\Delta \eta_{\mathrm{STH}, \mathrm{opt}}}{\Delta \eta_{\text {overpotential }}^{10 \mathrm{~mA} \mathrm{\textrm {cm } ^ { - 2 }}}}$, in general exhibited a very weak dependence on detailed kinetic parameters of the HER and OER or on the effective transport resistance of the membrane separator and the solution electrolyte. Leveraging the enhanced electrocatalysis and solution transport at elevated temperatures in the integrated system, the optimal STH conversion efficiency also exhibited a very weak dependence on the operational temperature. Using state-of-the-art catalyst systems, the optimal STH conversion efficiency showed a strong dependence on the optical concentration factor.

\section{Acknowledgements}

This material is based upon work performed by the Joint Center for Artificial Photosynthesis, a DOE Energy Innovation Hub, supported through the Office of Science of the U.S. Department of Energy under Award number DE-SC0004993.

\section{References}

1 M. G. Walter, E. L. Warren, J. R. McKone, S. W. Boettcher, Q. X. Mi, E. A. Santori and N. S. Lewis, Chem. Rev., 2010, 110, 6446-6473.

2 N. S. Lewis and D. G. Nocera, Proc. Natl. Acad. Sci. U. S. A., 2007, 104, 20142.

3 T. R. Cook, D. K. Dogutan, S. Y. Reece, Y. Surendranath, T. S. Teets and D. G. Nocera, Chem. Rev., 2010, 110, 64746502.

4 J. R. McKone, N. S. Lewis and H. B. Gray, Chem. Mater., 2014, 26, 407-414.

5 C. C. L. McCrory, S. Jung, J. C. Peters and T. F. Jaramillo, J. Am. Chem. Soc., 2013, 135, 16977-16987.

6 J. Suntivich, K. J. May, H. A. Gasteiger, J. B. Goodenough and Y. Shao-Horn, Science, 2011, 334, 1383-1385.

7 Q. X. Mi, Y. Ping, Y. Li, B. F. Cao, B. S. Brunschwig, P. G. Khalifah, G. A. Galli, H. B. Gray and N. S. Lewis, J. Am. Chem. Soc., 2012, 134, 18318-18324.

8 M. Long, W. M. Cai, J. Cai, B. X. Zhou, X. Y. Chai and Y. H. Wu, J. Phys. Chem. B, 2006, 110, 20211-20216.

9 A. Kudo, K. Omori and H. Kato, J. Am. Chem. Soc., 1999, 121, 11459-11467.

10 A. Kay, I. Cesar and M. Gratzel, J. Am. Chem. Soc., 2006, 128, 15714-15721.

11 B. Kumar, M. Llorente, J. Froehlich, T. Dang, A. Sathrum and C. P. Kubiak, Annu. Rev. Phys. Chem., 2012, 63, 541-569.

12 A. Saltelli, M. Ratto, T. Andres, F. Campolongo, J. Cariboni, D. Gatelli, M. Saisana and S. Tarantola, Global Sensitivity Analysis: The Primer, John Wiley \& Sons, 2008.
13 L. C. Seitz, Z. B. Chen, A. J. Forman, B. A. Pinaud, J. D. Benck and T. F. Jaramillo, ChemSusChem, 2014, 7, 1372-1385.

14 J. R. Bolton, S. J. Strickler and J. S. Connolly, Nature, 1985, 316, 495-500.

15 H. Doscher, J. Geisz, T. Deutsch and J. A. Turner, Energy Environ. Sci., 2014, 7, 2951-2956.

16 Y. K. Chen, C. X. Xiang, S. Hu and N. S. Lewis, J. Electrochem. Soc., 2014, 161(10), F1101-F1110.

17 S. Haussener, S. Hu, C. Xiang, A. Z. Weber and N. Lewis, Energy Environ. Sci., 2013, 6, 3605-3618.

18 S. Haussener, C. X. Xiang, J. M. Spurgeon, S. Ardo, N. S. Lewis and A. Z. Weber, Energy Environ. Sci., 2012, 5, 9922-9935.

19 S. Hu, C. X. Xiang, S. Haussener, A. D. Berger and N. S. Lewis, Energy Environ. Sci., 2013, 6, 2984-2993.

20 C. Xiang, Y. Chen and N. S. Lewis, Energy Environ. Sci., 2013, 6, 3713-3721.

21 C. C. L. McCrory, S. H. Jung, J. C. Peters and T. F. Jaramillo, J. Am. Chem. Soc., 2013, 135, 16977-16987.

22 J. L. Qiao, Y. Y. Liu, F. Hong and J. J. Zhang, Chem. Soc. Rev., 2014, 43, 631-675.

23 R. Bailis, M. Ezzati and D. M. Kammen, Science, 2005, 308, 98-103.

24 J. E. Campbell, G. R. Carmichael, T. Chai, M. Mena-Carrasco, Y. Tang, D. R. Blake, N. J. Blake, S. A. Vay, G. J. Collatz, I. Baker, J. A. Berry, S. A. Montzka, C. Sweeney, J. L. Schnoor and C. O. Stanier, Science, 2008, 322, 10851088.

25 J. M. Murphy, D. M. H. Sexton, D. N. Barnett, G. S. Jones, M. J. Webb and D. A. Stainforth, Nature, 2004, 430, 768-772. 26 W. Shockley and H. J. Queisser, J. Appl. Phys., 1961, 32, 510. 27 E. L. Warren, J. R. McKone, H. A. Atwater, H. B. Gray and N. S. Lewis, Energy Environ. Sci., 2012, 5, 9653-9661.

28 M. D. Kelzenberg, D. B. Turner-Evans, M. C. Putnam, S. W. Boettcher, R. M. Briggs, J. Y. Baek, N. S. Lewis and H. A. Atwater, Energy Environ. Sci., 2011, 4, 866-871.

29 M. C. Putnam, S. W. Boettcher, M. D. Kelzenberg, D. B. Turner-Evans, J. M. Spurgeon, E. L. Warren, R. M. Briggs, N. S. Lewis and H. A. Atwater, Energy Environ. Sci., 2010, 3, 1037-1041.

30 S. W. Boettcher, E. L. Warren, M. C. Putnam, E. A. Santori, D. Turner-Evans, M. D. Kelzenberg, M. G. Walter, J. R. McKone, B. S. Brunschwig, H. A. Atwater and N. S. Lewis, J. Am. Chem. Soc., 2011, 133, 1216-1219.

$31 \mathrm{~S} . \mathrm{Hu}, \mathrm{M}$. R. Shaner, J. A. Beardslee, M. Lichterman, B. S. Brunschwig and N. S. Lewis, Science, 2014, 344, 10051009.

32 A. J. Bard and L. R. Faulkner, Electrochemical Methods, Fundamentals and Applications, Wiley, 2nd edn, 2000.

33 K. Kinoshita, Electrochemical Oxygen Technology, Wiley, New York, 1992.

34 H. E. Darling, J. Chem. Eng. Data, 1964, 9, 421-426.

35 D. E. Brown, M. N. Mahmood, A. K. Turner, S. M. Hall and P. O. Fogarty, Int. J. Hydrogen Energy, 1982, 7, 405-410.

36 J. Landon, E. Demeter, N. Inoglu, C. Keturakis, I. E. Wachs, R. Vasic, A. I. Frenkel and J. R. Kitchin, ACS Catal., 2012, 2, 1793-1801. 
37 S. Trasatti, J. Electroanal. Chem., 1972, 39, 163.

38 J. McEnaney, J. Crompton, J. Callejas, E. Popczun, A. Biacchi, N. S. Lewis and R. Schaak, Chem. Mater., 2014, 26(16), 48264831.

39 J. M. McEnaney, J. C. Crompton, J. C. Callejas, E. J. Popczun, C. G. Read, N. S. Lewis and R. E. Schaak, Chem. Commun., 2014, 50, 11026-11028.

40 J. C. Callejas, J. M. McEnaney, C. G. Read, J. C. Crompton, A. J. Biacchi, E. J. Popczun, T. R. Gordon, N. S. Lewis and R. E. Schaak, ACS Nano, 2014, 8, 11101-11107.
41 Q. X. Mi, A. Zhanaidarova, B. S. Brunschwig, H. B. Gray and N. S. Lewis, Energy Environ. Sci., 2012, 5, 5694-5700.

42 J. M. Spurgeon, J. M. Velazquez and M. T. McDowell, Phys. Chem. Chem. Phys., 2014, 16, 3623-3631.

43 M. R. Singh, C. Xiang and N. S. Lewis, 2014, manuscript in preparation.

44 M. C. Hanna and A. J. Nozik, J. Appl. Phys., 2006, 100, 074510. 45 A. Berger, R. A. Segalman and J. Newman, Energy Environ. Sci., 2014, 7, 1468-1476.

46 Y. Hori, H. Wakebe, T. Tsukamoto and O. Koga, Electrochim. Acta, 1994, 39, 1833-1839. 